Preprint typeset in JHEP style. - HYPER VERSION

CERN-TH/2000-069

CITA-2000-06

hep-ph/0004024

February 29, 2000

\title{
Inflation After Preheating
}

\author{
Gary Felder \\ Department of Physics, Stanford University, Stanford, CA 94305, USA \\ and CITA, University of Toronto, 60 St George Str, Toronto, ON M5S 1A1, \\ Canada \\ E-mail: gfelder@leland.stanford.edu

\section{Lev Kofman} \\ CITA, University of Toronto, 60 St George Str, Toronto, ON M5S 1A1, Canada \\ E-mail: kofman@cita.utoronto.ca
}

\author{
Andrei Linde* \\ Theory Division, CERN CH 1211 Geneva 23, Switzerland \\ E-mail: 1inde@physics.stanford.edu \\ http://physics.stanford.edu/linde \\ Igor Tkachev \\ Institute for Theoretical Physics, ETH, Hönggerberg, CH-8093, Zurich, \\ Switzerland \\ E-mail: Igor.Tkachev@cern.ch
}

\begin{abstract}
Preheating after inflation may lead to nonthermal phase transitions with symmetry restoration. These phase transitions may occur even if the total energy density of fluctuations produced during reheating is relatively small as compared with the vacuum energy in the state with restored symmetry. As a result, in some inflationary models one encounters a secondary, nonthermal stage of inflation due to symmetry restoration after preheating. We review the theory of nonthermal phase transitions and make a prediction about the expansion factor during the secondary inflationary stage. We then present the results of lattice simulations which verify these predictions, and discuss possible implications of our results for the theory of formation of topological defects during nonthermal phase transitions.
\end{abstract}

KEYWORDS: cph.

\footnotetext{
${ }^{*}$ On leave of absence from Stanford University until 1 September 2000
} 


\section{Contents}

1. Introduction 1

2. Theory of the phase transition 2

3. Simulation Results and Their Interpretation 5

4. Nonthermal phase transitions and production of topological defects 11

5. Conclusions 13

6. Appendix: The Numerical Calculations 14

6.1 The Field Equations 15

6.2 The Scale Factor Equation 16

6.3 Initial Conditions 17

6.4 The Calculational Method: Staggered Leapfrog 19

\section{Introduction}

The theory of cosmological phase transitions is usually associated with symmetry restoration due to high temperature effects and the subsequent symmetry breaking which occurs as the temperature decreases in an expanding universe [1, 2, 3, 4]. A particularly important version of this theory is the theory of first order cosmological phase transitions developed in [3]. It served as a basis for the first versions of inflationary cosmology [5], as well as for the theory of electroweak baryogenesis [6].

Recently it was pointed out that preheating after inflation [7] may rapidly produce a large number of particles that for a long time remain in a state out of thermal equilibrium. These particles may lead to specific nonthermal cosmological phase transitions [8, 9]. In some cases these phase transitions are first order [10, 11]; they occur by the formation of bubbles of the phase with spontaneously broken symmetry inside the metastable symmetric phase. If the lifetime of the metastable state is large enough for the energy density of fluctuations to be diluted, one may encounter a short secondary stage of inflation after preheating [8]. Such a secondary inflation stage, if it occurs late enough, could be important in solving the moduli and gravitino problems. In this respect secondary "nonthermal" inflation due to preheating 
may be an alternative to the "thermal inflation" [12], suggested for solving these problems.

In this paper we will briefly present the theory of such phase transitions and then give the results of numerical lattice simulations that directly demonstrated the possibility of such brief inflation. We will also discuss possible implications of our results for the theory of formation of topological defects during nonthermal phase transitions. A detailed description of numerical methods used in our work will be given in the appendix.

\section{Theory of the phase transition}

Consider a set of scalar fields with the potential

$$
V(\phi, \chi)=\frac{\lambda}{4}\left(\phi^{2}-v^{2}\right)^{2}+\frac{g^{2}}{2} \phi^{2} \chi^{2} .
$$

The inflaton field $\phi$ has a double-well potential and interacts with an $N$-component scalar field $\chi ; \chi^{2} \equiv \sum_{i=1}^{N} \chi_{i}^{2}$. For simplicity, the field $\chi$ is taken to be massless and without self-interaction. The fields couple minimally to gravity in a FRW universe with a scale factor $a(t)$.

The potential $V(\phi, \chi)$ has minima at $\phi= \pm v, \chi=0$ and a local maximum in the $\phi$ direction at $\phi=\chi=0$ with curvature $V_{, \phi \phi}=-\lambda v^{2}$. The effective potential acquires corrections due to quantum and/or thermal fluctuations of the scalar fields [迫, 目, 田],

$$
\Delta V=\frac{3}{2} \lambda\left\langle\phi^{2}\right\rangle \phi^{2}+\frac{g^{2}}{2}\left\langle\chi^{2}\right\rangle \phi^{2}+\frac{g^{2}}{2}\left\langle\phi^{2}\right\rangle \chi^{2}+\ldots,
$$

where we have written only the leading terms depending on $\phi$ and $\chi$. The effective mass squared of the field $\phi$ is given by

$$
m_{\phi}^{2}=-m^{2}+3 \lambda \phi^{2}+3 \lambda\left\langle\phi^{2}\right\rangle+g^{2}\left\langle\chi^{2}\right\rangle,
$$

where $m^{2}=\lambda v^{2}$. Symmetry is restored, i.e. $\phi=0$ becomes a stable equilibrium point, when the fluctuations $\left\langle\phi^{2}\right\rangle,\left\langle\chi^{2}\right\rangle$ become sufficiently large to make the effective mass squared positive at $\phi=0$.

For example, one may consider matter in thermal equilibrium. Then, in the large temperature limit, one has $\left\langle\phi^{2}\right\rangle=\left\langle\chi_{i}^{2}\right\rangle=\frac{T^{2}}{12}$. The effective mass squared of the field $\phi$

$$
m_{\phi, e f f}^{2}=-m^{2}+3 \lambda \phi^{2}+3 \lambda\left\langle\delta \phi^{2}\right\rangle+g^{2}\left\langle\chi^{2}\right\rangle
$$

is positive and symmetry is restored (i.e. $\phi=0$ is the stable equilibrium point) for $T>T_{c}$, where $T_{c}^{2}=\frac{12 m^{2}}{3 \lambda+N g^{2}} \gg m^{2}$. At this temperature the energy density of the gas of ultrarelativistic particles is given by

$$
\rho=\mathcal{N}\left(T_{c}\right) \frac{\pi^{2}}{30} T_{c}^{4}=\frac{24 m^{4} \mathcal{N}\left(T_{c}\right) \pi^{2}}{5\left(3 \lambda+N g^{2}\right)^{2}} .
$$


Here $\mathcal{N}(T)$ is the effective number of degrees of freedom at large temperature, which in realistic situations may vary from $10^{2}$ to $10^{3}$. We will assume that $N g^{2} \gg \lambda$, see below. For $g^{4}<\frac{96 \mathcal{N}\left(T_{c}\right) \pi^{2}}{5 N^{2}} \lambda$ the thermal energy at the moment of the phase transition is greater than the vacuum energy density $V(0)=\frac{m^{4}}{4 \lambda}$, which means that the phase transition does not involve a stage of inflation.

In fact, the phase transition with symmetry breaking occurs not at $T>T_{c}$, but somewhat earlier [3]. To understand this effect let us compare the temperature $T_{c} \sim m /(\sqrt{N} g)$ and the mass $m_{\chi}=g \phi$ of the $\chi$ particles in the minimum of the zero-temperature effective potential at $\phi=v=m / \sqrt{\lambda}$. One can easily see that $m_{\chi} \gg T_{c}$ for $N g^{4} \gg \lambda$. This means that for $N g^{4} \gg \lambda$ the temperature $T_{c}$ is insufficient to excite perturbations of the fields $\chi_{i}$ at $\phi=v$. As a result, these perturbations do not change the shape of the effective potential $\phi=v$. Thus the potential at $T$ slightly above $T_{c}$ has its old zero-temperature minimum at $\phi=v$, as well as the temperature-induced minimum at $\phi=0$. Symmetry breaking occurs as a first-order phase transition due to formation of bubbles of the phase with $\phi \approx v$ at some temperature above $T_{c}$ when the minimum at $\phi=v$ becomes deeper than the minimum at $\phi=0$, and the probability of bubble formation becomes sufficiently large. A more detailed investigation in the case $N=1$ shows that the phase transition is first order under a weaker condition $g^{3} \gg \lambda$ [3].

In the case $N g^{4}>10^{2} \lambda$ the phase transition occurs after a secondary stage of inflation. In this regime radiative corrections are important. They lead to the creation of a local minimum of $V(\phi, \chi)$ at $\phi=0$ even at zero temperature, and the phase transition occurs from a strongly supercooled state [3]. That is why the first models of inflation required supercooling at the moment of the phase transition [5].

In supersymmetric theories one may have $N g^{4} \gg 10^{2} \lambda$ and still have a potential which is flat near the origin due to cancellation of quantum corrections of bosons and fermions [12]. In such cases the thermal energy becomes smaller than the vacuum energy at $T<T_{0}$, where $T_{0}^{4}=\frac{15}{2 \mathcal{N} \pi^{2}} m^{2} v^{2}$. Then one may have a short stage of inflation which begins at $T \sim T_{0}$ and ends at $T=T_{c}$. During this time the universe may inflate by the factor

$$
\frac{a_{c}}{a_{0}}=\frac{T_{0}}{T_{c}} \sim 10^{-1}\left(\frac{g^{4}}{\lambda}\right)^{1 / 4} .
$$

Similar phase transitions may occur much more efficiently prior to thermalization, due to the anomalously large fluctuations $\left\langle\phi^{2}\right\rangle$ and $\left\langle\chi^{2}\right\rangle$ produced during preheating [8, 9]. These fluctuations can change the shape of the effective potential and lead to symmetry restoration. Afterwards, the universe expands, the values of $\left\langle\phi^{2}\right\rangle$ and $\left\langle\chi^{2}\right\rangle$ drop down, and the phase transition with symmetry breaking occurs.

An interesting feature of nonthermal phase transitions is that they may occur even in theories where the usual thermal phase transitions do not happen. The main reason can be understood as follows. Suppose reheating occurs due to the decay of a scalar field with energy density $\rho$. If this energy is instantly thermalized, 
then one obtains relativistic particles with energy density $O\left(T^{4}\right)$ which in the first approximation can be represented as $\rho \approx E^{2}\left(\left\langle\phi^{2}\right\rangle+\left\langle\chi^{2}\right\rangle\right)$. Here $E \sim T \sim \rho^{1 / 4}$ is a typical energy of a particle in thermal equilibrium. After preheating, however, one has particles $\phi$ and $\chi$ with much smaller energy but large occupation numbers. As a result, the same energy release may create much greater values of $\left\langle\phi^{2}\right\rangle$ and $\left\langle\chi^{2}\right\rangle$ than in the case of instant thermalization. This may lead to symmetry restoration after preheating even if the symmetry breaking occurs on the GUT scale, $v \sim 10^{16}$ $\mathrm{GeV}$ [8, 9].

The main conclusions of [8, 9] have been confirmed by detailed investigation using lattice simulations in [10, 13, 14, 11]. One of the main results obtained in [10] was that for sufficiently large $g^{2}$ nonthermal phase transitions are first order. They occur from a metastable vacuum at $\phi=0$ due to the creation of bubbles with $\phi \neq 0$. This result is very similar to the analogous result in the theory of thermal phase transitions [3]. According to [10], the necessary conditions for this transition to occur and to be of the first order can be formulated as follows:

(i) At the time of the phase transition, the point $\phi=0$ should be a local minimum of the effective potential. From (2.3), we see that this means that $N g^{2}\left\langle\chi_{i}^{2}\right\rangle>\lambda v^{2}$.

(ii) At the same time, the typical momentum $p_{*}$ of $\chi_{i}$ particles should be smaller than $g v$. This is the condition of the existence of a potential barrier. Particles with momenta $p<g v$ cannot penetrate the state with $|\phi| \approx v$, so they cannot change the shape of the effective potential at $|\phi| \approx v$. Therefore, if both conditions (i) and (ii) are satisfied, the effective potential has a local minimum at $\phi=0$ and two degenerate minima at $\phi \approx \pm v$.

(iii) Before the minima at $\phi \approx \pm v$ become deeper than the minimum at $\phi=0$, the inflaton's zero mode should decay significantly, so that it performs small oscillations near $\phi=0$. Then, after the minimum at $|\phi| \approx v$ becomes deeper than the minimum at $\phi=0$, fluctuations of $\phi$ drive the system over the potential barrier, creating an expanding bubble.

The investigation performed in [10] confirmed that for sufficiently large $g^{2}$ and $N$ these conditions are indeed satisfied and the phase transition is first order. One may wonder whether for $g^{2} \gg \lambda$ one may have a stage of inflation in the metastable vacuum $\phi=0$.

Analytical estimates of Ref. [8] suggested that this is indeed the case, and the degree of this inflation for $N=1$ is expected to be

$$
\frac{a_{c}}{a_{0}} \sim\left(\frac{g^{2}}{\lambda}\right)^{1 / 4}
$$

which is much greater than the number $10^{-1}\left(\frac{g^{4}}{\lambda}\right)^{1 / 4}$ in the thermal inflation scenario. One could also expect that the duration of inflation, just like the strength of the phase transition, increases if one considers $\mathrm{N}$ fields $\chi_{i}$ with $N \gg 1$. 
However, the theory of preheating is extremely complicated, and there are some factors which could not be adequately taken into account in the simple estimates of [8]. The most important factor is the effect of rescattering of particles produced during preheating [15]. This effect tends to shut down the resonant production of particles and thus shorten or prevent entirely the occurrence of a secondary stage of inflation. Thus the estimates above reflect the maximum degree of inflation possible for a given set of parameter values, but in practice the expansion factor will be somewhat smaller than these predictions. The only way to fully account for all the effects of backreaction and expansion is through numerical lattice simulations. In our paper we used a generalized version of the method of lattice simulations developed in [15, 10]. In the next section we will describe the basic features of our method and describe our main results. A detailed description of the lattice simulations will be given in the appendix.

\section{Simulation Results and Their Interpretation}

In our paper we will take $\lambda \approx 10^{-13}$, which gives the proper magnitude of inflationary perturbations of density [4, [16]. We assume that $g^{2} \gg \lambda$, and consider $v \approx 10^{16} \mathrm{GeV}$, which corresponds to the GUT scale. A numerical investigation of preheating in the model (2.1) was first performed in [10]. The authors found a strongly first order phase transition. The strength of the phase transition increased with an increase of $g^{2} / \lambda$ and of the number $N$ of the fields $\chi_{i}$. However, for the parameters of the model studied in $[10]\left(g^{2} / \lambda \approx 200\right)$ there was no inflation during symmetry restoration. This is not unexpected because the estimates discussed above indicated that the expansion of the universe during the short stage of nonthermal inflation cannot be greater than $\left(\frac{g^{2}}{\lambda}\right)^{1 / 4}$.

Keeping in mind that $\lambda$ in this model is extremely small, one would expect that in realistic versions of this model one may have $g^{2} / \lambda$ as large as $10^{10}$, which could lead to a relatively long stage of inflation. However, for very large $g^{2} / \lambda$ our analytical estimates are unreliable, and lattice simulations become extremely difficult: One needs to have enormously large lattices to keep both infrared and ultraviolet effects under control.

To mimic the effects of large $g^{2}$, we considered a large number of the fields $\chi_{i}$. We have performed simulations for $g^{2} / \lambda=800$ and $N=19$. With these parameters the strength of the phase transition became much greater, and there was a short stage of inflation prior to the phase transition. The details of our calculation and an explanation of our methods are given in the appendix. Here we only present the main results.

The simulation showed that the oscillations of the inflaton field decreased until

the field was trapped near zero. It remained there until the moment of the phase transition when it rapidly jumped to its symmetry breaking value, as shown in Fig. 1. 


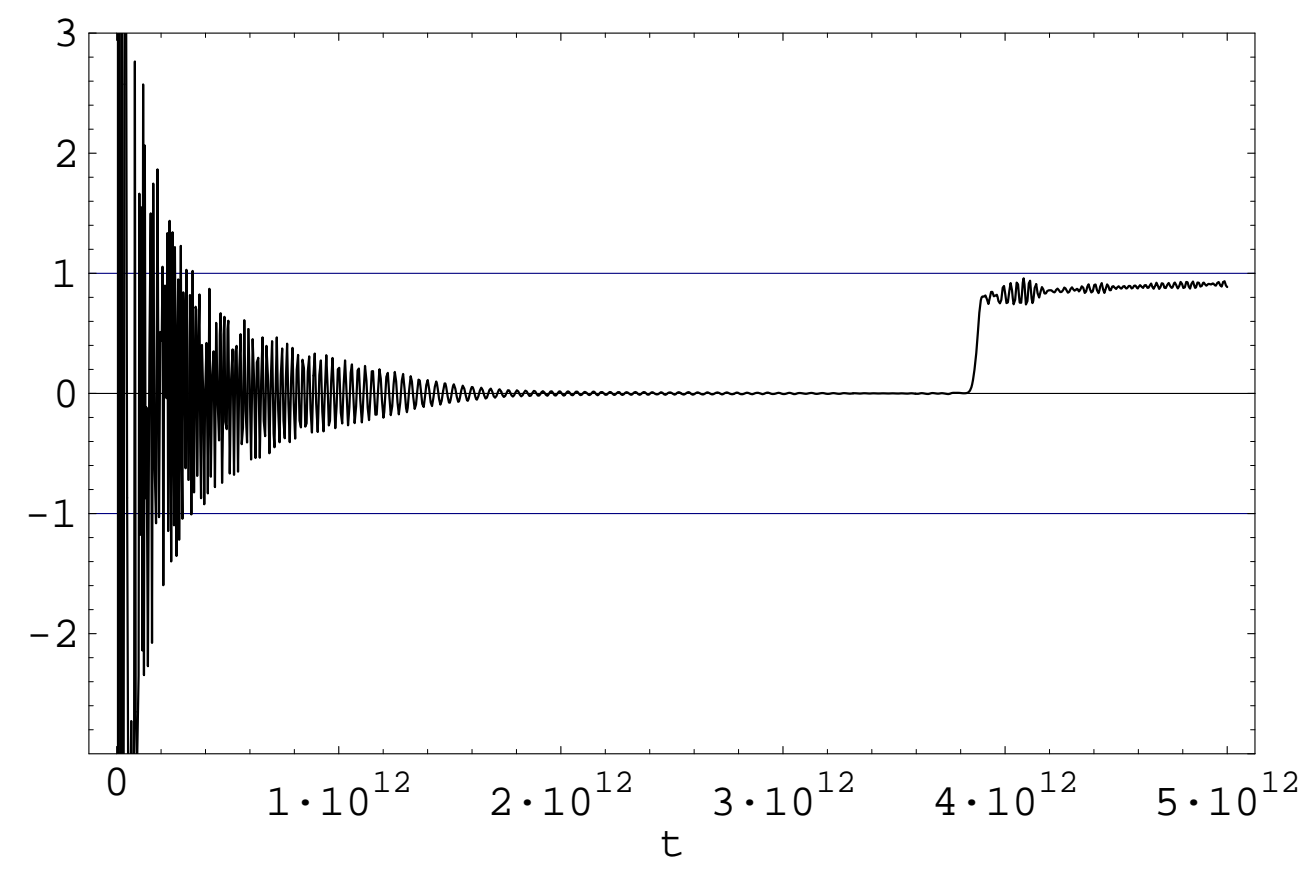

Figure 1: The spatial average of the inflation field $\phi$ as a function of time. The field $\phi$ is shown in units of $v$, the symmetry breaking parameter. Time is shown in Planck units.

The trapping of the field occurred because of the corrections to the effective potential induced by the particles $\phi$ and $\chi$ produced during preheating, just like in the theory of high-temperature phase transitions. In our case, however, this effect has some unusual features.

To first order in $g^{2}$, the leading contribution to the equation of motion $\ddot{\phi}=-V^{\prime}$ is given by $g^{2} \phi\left\langle\chi^{2}\right\rangle$, where

$$
\left\langle\chi^{2}\right\rangle \approx \frac{N}{2 \pi^{2}} \int_{0}^{\infty} \frac{n_{k} k^{2} d k}{\omega_{k}(\phi)} .
$$

Here $\omega_{k}=\sqrt{k^{2}+g^{2}\left(\phi^{2}+\left\langle\phi^{2}\right\rangle\right)}$ is the energy of $\chi_{i}$ particles with momentum $k$ and $n_{k}$ is their occupation number; $\phi$ is the homogeneous component of the field. For $\phi \ll \sqrt{\left\langle\phi^{2}\right\rangle}$, one has

$$
\left\langle\chi^{2}\right\rangle_{\phi=0} \approx \frac{N}{2 \pi^{2}} \int_{0}^{\infty} \frac{n_{k} k^{2} d k}{\sqrt{k^{2}+g^{2}\left\langle\phi^{2}\right\rangle}} .
$$

This quantity does not depend on $\phi$; it can be evaluated using our lattice simulations when the field $\phi$ oscillates near $\phi=0$. It leads to the usual quadratic correction to the effective potential, see Eq. (2.2). This correction adequately describes the change of the shape of the effective potential for $\phi$ smaller than the amplitude of the oscillations of this field, because most of the time prior to the moment of the phase transition this amplitude is much smaller than $\sqrt{\left\langle\phi^{2}\right\rangle}$. 
However, if we want to evaluate the effective potential at all values of $|\phi|$ from 0 to $v$, rather than for $\phi$ similar to the amplitude of the oscillations, then one should take into account that for sufficiently large $|\phi|$ the term $g|\phi|$ becomes greater than $g \sqrt{\left\langle\phi^{2}\right\rangle}$ and than the typical momentum $k$ of particles $\chi_{i}$. In this case the main contribution to $\left\langle\chi^{2}\right\rangle$ is given by nonrelativistic particles with $\omega_{k} \approx+g|\phi|$, and one has

$$
\left\langle\chi^{2}\right\rangle \approx \frac{N}{2 \pi^{2}} \int_{0}^{\infty} \frac{n_{k} k^{2} d k}{g|\phi|}=\frac{n_{\chi}}{g|\phi|},
$$

where $n_{\chi}$ is the total density of all types of $\chi_{i}$ particles. This implies that at large $|\phi|$ the effective potential acquires a correction

$$
\delta V \approx g|\phi| n_{\chi}
$$

Thus, instead of being quadratic or cubic in $|\phi|$, as one could expect from the analogy with the high-temperature theory [3, 11], the corrections to the effective potential at large $|\phi|$ are proportional to $|\phi|[7]$.

The combination of these two types of corrections to the effective potential (quadratic at small $|\phi|$ and linear at large $|\phi|$ ) leads to the symmetry restoration that we have found in our lattice simulations.

It is instructive to look in a more detailed way at the small region near the time of the phase transition. The first of the graphs in Fig. 2 shows the oscillations of the field $\phi$ soon before the phase transition, whereas the second one shows these oscillations soon afterwards.
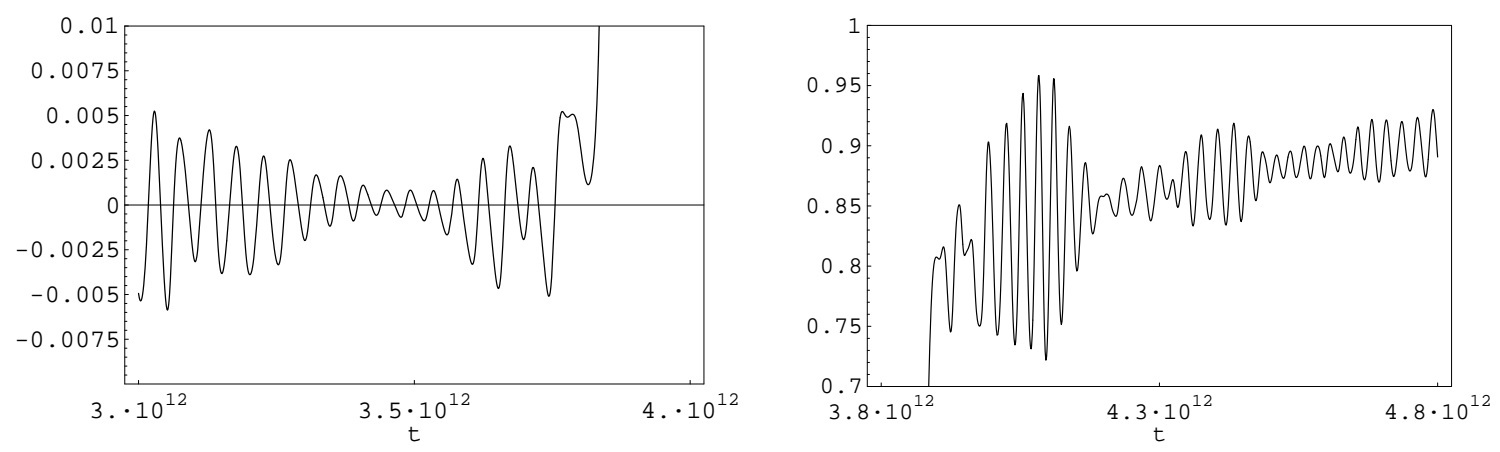

Figure 2: The spatial average of the inflation field $\phi$ as a function of time in the vicinity of the phase transition. The left figure shows the field just before the phase transition and at the moment of the transition. The field oscillates with an amplitude approaching $10^{-3} v$. The right figure shows the field $\phi$ after the phase transition, when it oscillates near the (time-dependent) position of the minimum of the effective potential at $\phi \approx v$. Time is shown in Planck units. 
First of all, one can see that just before the phase transition the field oscillates with an amplitude three orders of magnitude smaller than $v$, which is a clear sign of symmetry restoration. Another interesting feature is that the frequency of oscillations does not vanish as we approach the phase transition, but remains nearly constant. Moreover, this frequency is only about two times smaller than the frequency of oscillations after the phase transition, which is equal to $\sqrt{2} m$. Note that the frequency of the oscillations is determined by the effective mass of the scalar field, which is given by the curvature of the effective potential: $m_{\phi}^{2}=V^{\prime \prime}$. This means that at the moment of the phase transition the effective potential has a deep minimum at $\phi=0$ with curvature $V^{\prime \prime} \sim+m^{2}$, i.e. the phase transition is strongly first order. Such phase transitions should occur due to the formation of bubbles containing nonvanishing field $\phi$.

Indeed, we have found that this transition occurred in a nearly spherical region of the lattice that quickly grew to encompass the entire space. The growth of this region of the new phase is shown in Fig. 3. The nearly perfect sphericity of this region is an additional indication that the transition was strongly first-order. In comparison, the bubble observed in the lattice simulations of [10] for $g^{2} / \lambda \approx 200$ was not exactly spherically symmetric.

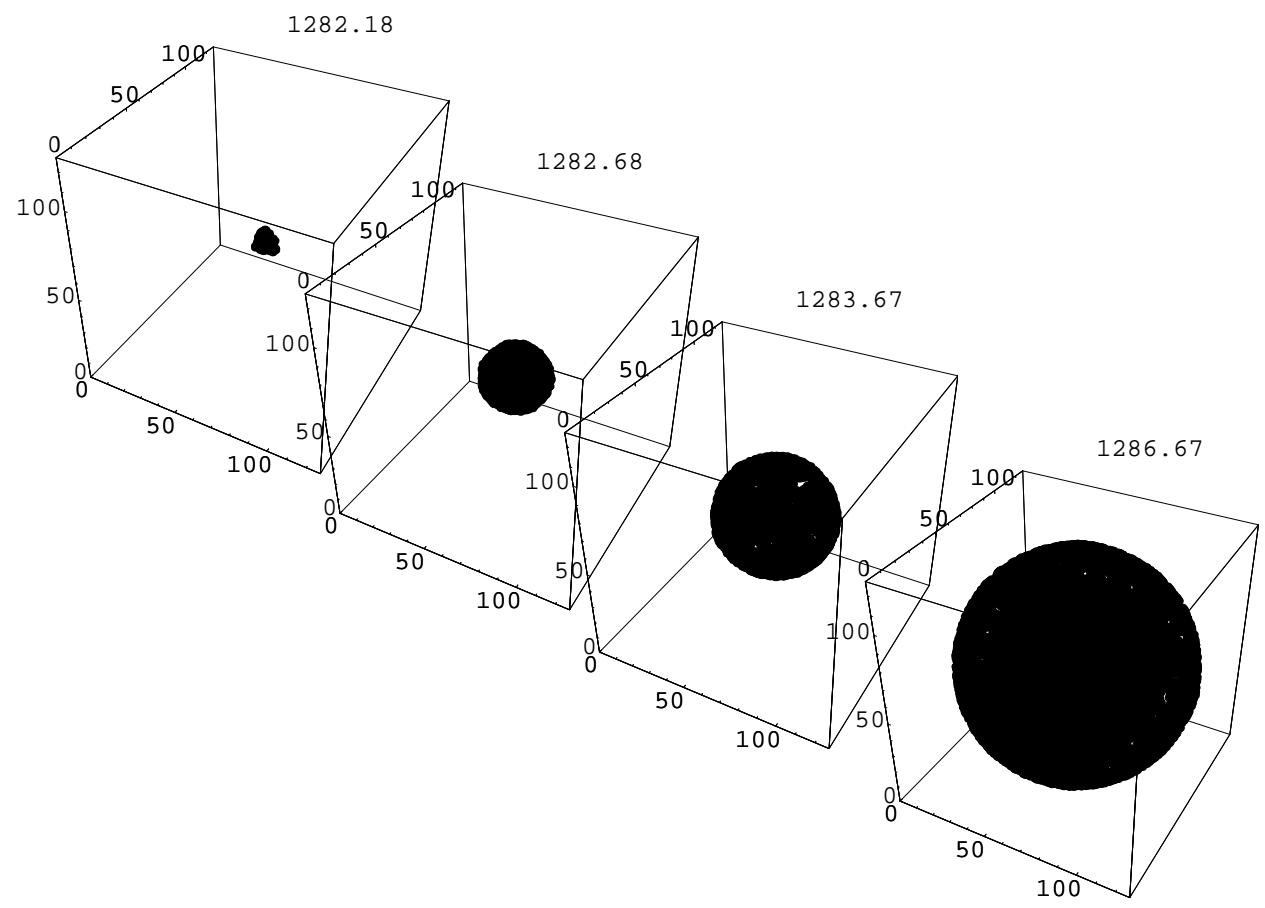

Figure 3: These plots show the region of space in which symmetry breaking has occurred at four successive times labeled by the value of the conformal time $t_{p r}$.

The first order phase transition and bubble formation seen in our simulations can be understood as a result of gradual accumulation of classical fluctuations $\delta \phi(t, \vec{x})$. 
These fluctuations stochastically climb up from $\phi=0$ towards the local maximum $\phi_{*}$ of the effective potential. Consider the regions in which $\delta \phi(t, \vec{x})>\phi_{*}$. If the probability of formation of such regions is small because they correspond to high peaks of the random field $\phi$, then these regions will have a nearly spherical shape and can be represented by spherical surfaces of radius $R_{*}$ (bubbles). If the radius $R_{*}$ is small, gradient terms will prevent the field $\phi$ inside the region from rolling down towards the global minimum at $\phi=v$ (subcritical bubble). If $R_{*}$ is large enough, the gradient terms cannot push the field back to the metastable state $\phi=0$ and the field inside the bubble rolls towards the global minimum, forming a bubble of ever increasing radius. This process can be described within the stochastic approach to tunneling proposed in [17]. Typically, the gradient terms cannot win over the potential energy terms if $R_{*}>O\left(\left|m_{\phi}^{-1}\right|\right)$, where $m_{\phi}$ corresponds to the effective mass of the scalar field in the interior of the bubble. This provides an estimate for the initial size of the bubble $R_{*} \sim O\left(\left|m_{\phi}^{-1}\right|\right)$.

The phase transition occurs from a state with energy density dominated by the vacuum energy density $V(0)$. Figure 4 shows the scale factor $a$ as a function of time. The curvature becomes slightly positive at the time before the phase transition, which indicates a short stage of exponential growth of the universe. Because the curvature is hard to see in figure 4 we have also plotted the second derivative $\ddot{a}$ in figure 5. While the inflaton is trapped in the false vacuum state, $\ddot{a}$ becomes positive, indicating a brief stage of inflation.

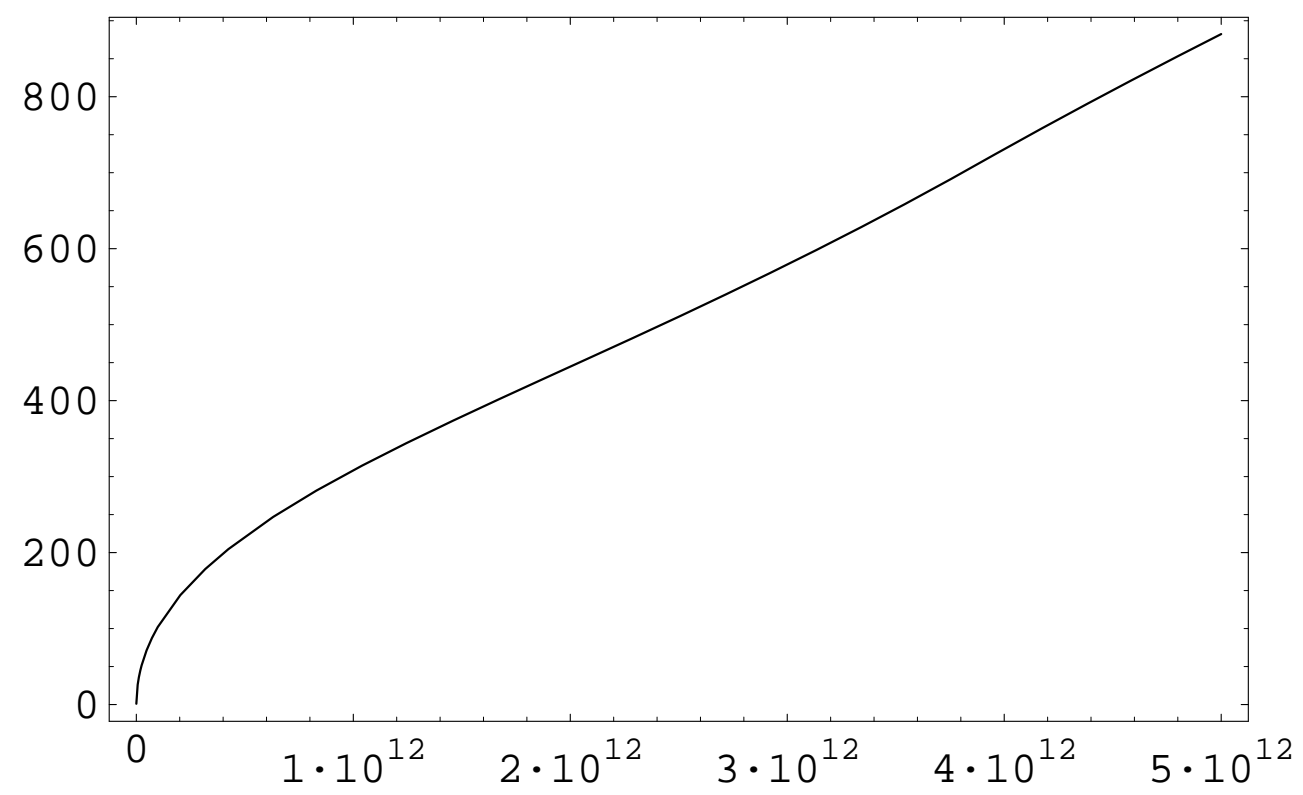

Figure 4: The scale factor $a$ as a function of time. In the beginning $a \sim \sqrt{t}$, which is a curve with negative curvature, but then at some stage it begins to turn upwards, indicating a short stage of inflation. 


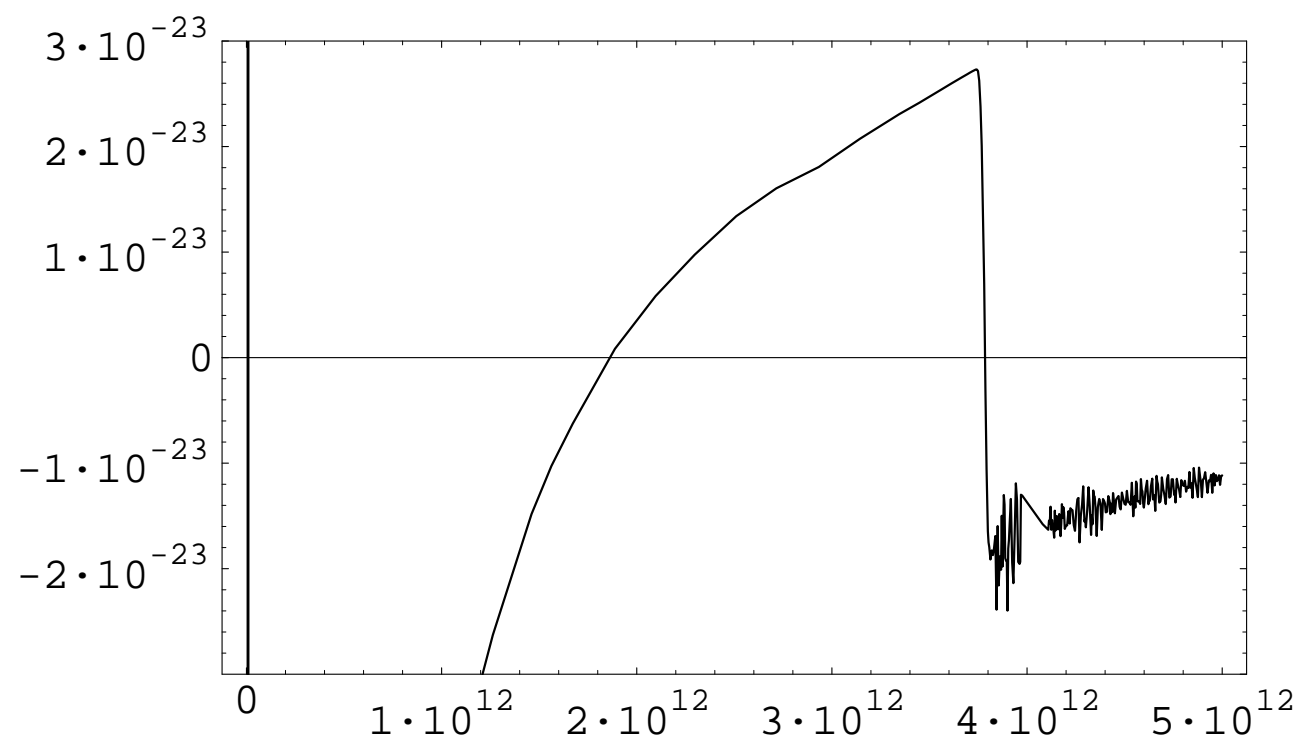

Figure 5: The second derivative of the scale factor, $\ddot{a}$. A universe dominated by ordinary matter (relativistic or nonrelativistic) will always have $\ddot{a}<0$, whereas in an inflationary universe $\ddot{a}>0$. We see that starting from the moment $t \sim 2 \times 10^{12}$ (in Planck units) the universe experiences accelerated (inflationary) expansion.

Another signature of inflation is an equation of state with negative pressure. Figure 6 shows the parameter $\alpha=p / \rho$, which becomes negative during the metastable phase. At the moment of the phase transition the universe becomes matter dominated and the pressure jumps to nearly 0 .

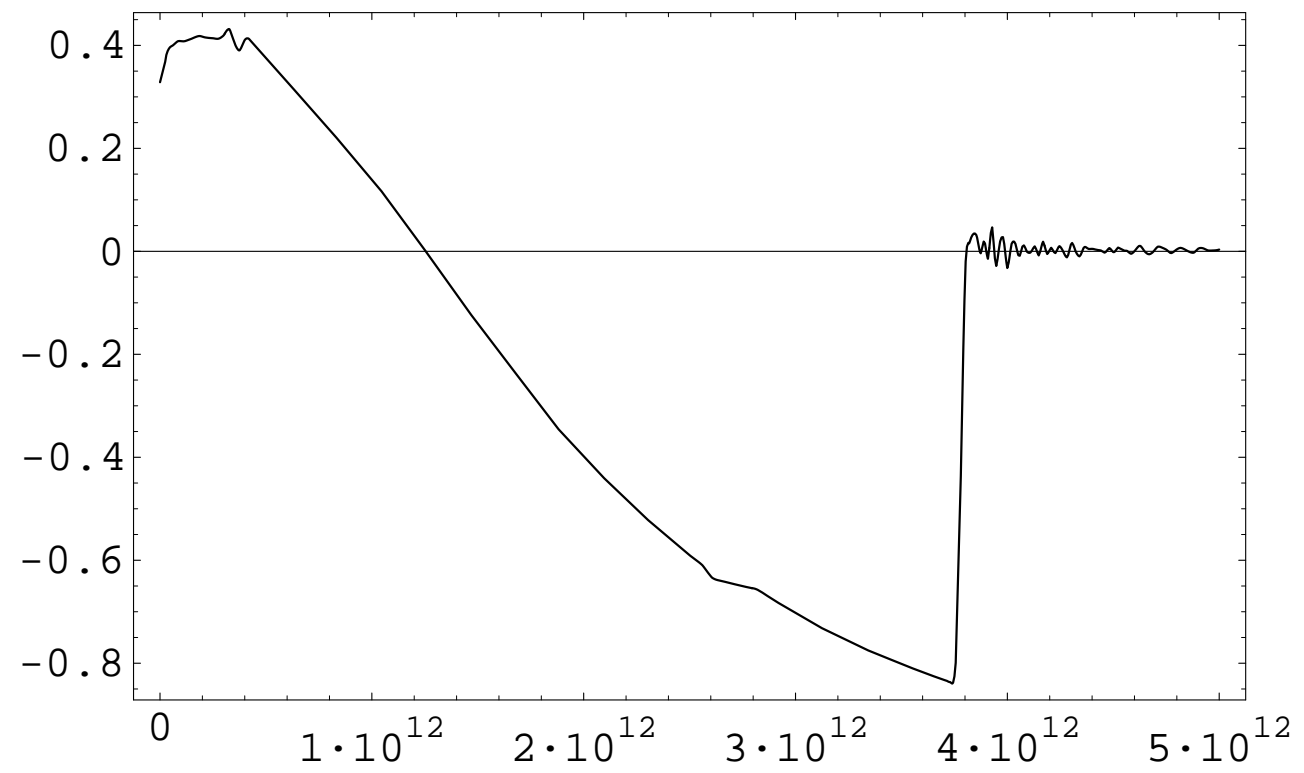

Figure 6: The ratio of pressure to energy density $p / \rho$. (Values were time averaged over short time scales to make the plot smoother and more readable.) 
From the beginning of this inflationary stage (roughly when the pressure becomes negative) to the moment of the phase transition the total expansion factor is 2.1. As expected this is of the same order but somewhat lower than the predicted maximum, $\left(\frac{g^{2}}{\lambda}\right)^{1 / 4} \approx 5.3$. We can thus conclude that it is possible to achieve inflation for parameters for which this would not have been possible in thermal equilibrium $\left(g^{4} \ll\right.$ $\lambda)$. In our simulation we have shown the occurrence of a very brief stage of inflation. This stage may be much longer for larger (realistic) values of $g^{2} / \lambda$. However, to check whether this is indeed the case one would need to perform a more detailed investigation on a lattice of a much greater size.

\section{Nonthermal phase transitions and production of topologi- cal defects}

In this section we would like to discuss possible implications of our investigation for the theory of production of topological defects after preheating [10, 13, 14].

The bubbles that appear after the phase transition can contain either positive or negative field, $\phi= \pm v$. If bubbles of either type are formed with comparable probability, then after the phase transition the universe becomes divided into nearly equal numbers of domains with $\phi= \pm v$, separated by domain walls. Such domain walls would lead to disastrous cosmological consequences, which would rule out the models where this may happen [8, 9].

In general, the number of bubbles with $\phi=+v$ may be much greater (or much smaller) than the number of bubbles with $\phi=-v$. Then the domain wall problem does not appear because the bubbles with $\phi=+v$ would rapidly eat all their competitors with $\phi=-v$ (or vice versa). This may happen, for example, if the moment of the bubble production is determined by the coherently oscillating scalar field $\phi$. In such a case, after oscillating a bit near the top of the effective potential, the field $\phi$ may wind up in the same minimum of the effective potential everywhere in the universe.

To investigate the domain wall problem in our model one would need to repeat the calculation many times with slightly different initial conditions or to make them in a box of a much greater size that would allow one to see many bubbles simultaneously. Fortunately, the results obtained in our study may be sufficient to give an answer to this question without extremely large simulations.

First of all, according to the stochastic approach [17] to the theory of tunneling with bubble formation [18, 19], the bubbles of the field $\phi$ are created as a result of the accumulation of long-wavelength fluctuations of the scalar field with momenta $k$ smaller than the typical mass scale $m_{\phi}$ associated with this field, see Section 3. In our case this mass scale is related to the frequency of oscillations of the scalar field 
at the moment of the phase transition. At that moment the leading contribution to the fluctuations $\left\langle\phi^{2}\right\rangle$ is given by fluctuations with momenta much smaller than $m_{\phi}$. We calculated the value of the long-wavelength component of $\sqrt{\left\langle\phi^{2}\right\rangle}$ and found that it is (approximately) of the same order as the amplitude of oscillations of the field $\phi$ at the moment of the phase transition. The existence of a first-order phase transition suggests that the probability of bubble formation must have been exponentially suppressed during the metastable stage. Such suppression would only occur only if the amplitude of fluctuations required to form a bubble of the new phase was much larger than $\sqrt{\left\langle\phi^{2}\right\rangle}$ [17], which would in turn mean the required amplitude was much greater than the amplitude of oscillations of $\phi$. This suggests that the probability of the bubble formation is almost entirely determined by the incoherent fluctuations of the field $\phi$ rather than by the small coherent oscillations of this field. Consequently, the probability of formation of bubbles containing $\phi=+v$ in the first approximation must be equal to the probability of formation of bubbles containing $\phi=-v$.

To make this statement more reliable one would need to estimate the amplitude of the long-wavelength fluctuations of the field $\phi$ in a more precise way, which would involve using lattices of a greater size. However, there is additional evidence suggesting that the number of bubbles with positive and negative $\phi$ must be approximately equal to each other.

Indeed, as we have seen, the curvature of the effective potential remained approximately constant during dozens of oscillations of the field $\phi$ prior to the moment of the phase transition. This suggests that the shape of the effective potential and, consequently, the probability of the tunneling, did not change much during a single oscillation. Therefore one may expect that, within a single oscillation, the probability of a bubble forming when the oscillating field $\phi$ was negative was approximately the same as the probability of the bubble forming when it was positive.

If the number of bubbles with positive and negative $\phi$ is approximately equal to each other, the phase transition leads to the formation of dangerous domain walls, which rules out our model [20]. If correct, this is a rather important conclusion which shows that the investigation of nonthermal phase transition may rule out certain classes of inflationary models which otherwise would seem quite legitimate [8, 9].

But this conclusion does not imply that all theories where the nonthermal phase transition is strongly first order are ruled out. For example, one may consider a model (2.1) with $\phi$ being not a real but a complex field, $\phi=\frac{1}{\sqrt{2}}\left(\phi_{1}+i \phi_{2}\right),|\phi|^{2}=\frac{1}{2}\left(\phi_{1}^{2}+\phi_{2}^{2}\right)$. Since the main contribution to the effective potential of the field $\phi$ in the theory (2.1) is given not by the field(s) $\phi$ but by the fields $\chi_{i}$, we expect that this generalization will not lead to a qualitative modification of our results. In particular, we expect that for sufficiently large $N$ and $g^{2} / \lambda$ the phase transition will be strongly first order and there will be a short stage of inflation after preheating. However, in the new 
model we will have strings instead of domain walls.

A similar model in the absence of interaction of the fields $\phi$ with the fields $\chi$ was studied in [13, 14]. It was argued that even in this case infinite strings may be formed. The theory of galaxy formation due to cosmic strings is currently out of favor, but it is certainly true that cosmic strings produced after inflation may add new interesting features to the standard theory of formation of the large-scale structure of the universe 21]

The possibility of strongly first order phase transitions induced by preheating in models with $g^{2} \gg \lambda$ adds new evidence that infinite strings can be produced after nonthermal phase transitions. Indeed, infinite strings may not be produced if the direction in which the field $\phi$ falls from the point $\phi=0$ at the moment of the phase transition is determined by the oscillations of the field $\phi$. If, just as in the case

discussed above, the amplitude of these oscillations are much smaller than $\sqrt{\left\langle\phi^{2}\right\rangle}$ at the moment of the phase transition, then infinite strings are indeed formed.

\section{Conclusions}

The results of our lattice simulation confirm our expectations that preheating may lead to nonthermal phase transitions even in those theories where spontaneous symmetry breaking occurs at the GUT scale, $v \sim 10^{16} \mathrm{GeV}$. Some time ago this question was intensely debated in the literature. Some authors claimed that nonthermal phase transitions induced by preheating are impossible, and the notion of the effective potential after preheating is useless. In our opinion, Figs. 1, 2 and 3 give a clear answer to this question. In particular, Fig. 1 shows that $90 \%$ of the time from the end of inflation to the moment of symmetry breaking the field $\phi$ oscillates about $\phi=0$ with an amplitude much smaller than $v$. This could happen only because the corrections to the effective potential induced by particles $\phi$ and $\chi$ change the shape of $V(\phi)$ near $\phi=0$, turning its maximum into a deep local minimum.

In some theories, this effect may lead to production of superheavy strings, which may have important cosmological implications for the theory of formation of the large scale structure of the universe. In some other theories, these phase transitions may lead to excessive production of monopoles and domain walls. This may rule out a broad class of otherwise acceptable inflationary models.

In this paper we have shown that under certain conditions a nonthermal phase transition may lead to a short secondary stage of inflation. It would be interesting to study the possibility that a secondary stage of inflation induced by preheating could help solve the moduli and gravitino problems. The answer to this question will be strongly model-dependent because gravitinos can be produced by the oscillating scalar field even after the secondary inflation [22, 23]. Independently of all practical implications, the possibility of a secondary stage of inflation induced by preheating 
seems very interesting because it clearly demonstrates the potential importance of nonperturbative effects in post-inflationary cosmology.

The authors are grateful to Julian Borrill for very useful discussions. This work was supported by NSERC and CIAR and by NSF grant AST95-29-225. The work of G.F. and A.L. was also supported by NSF grant PHY-9870115, and the work of L.K. and A.L. by NATO Linkage Grant 975389.

\section{Appendix: The Numerical Calculations}

Our lattice program solves the classical equations of motion for a set of scalar fields in a Friedman-Robertson-Walker universe. These fields include an inflaton $\phi$ coupled to a set of matter fields $\chi_{i}$. The scale factor $a$ is also solved for self-consistently. In this appendix we describe the exact equations being solved and the method used to solve them.

Unless otherwise specified, all variables names refer to their bare physical values measured in Planck units. The quantities as they appear in the program have been rescaled in several ways, and these values will be indicated with $p r$, as in $\phi_{p r}$. The relations between the rescaled variables and their bare values are given below. Also, when we want to refer to a general property of the fields $\phi$ and $\chi_{i}$ we will use $\sigma$ to indicate a generic scalar field.

The simulations we discuss here used a grid of $128^{3}$ points. The spacing of the points was chosen so as to ensure that the ultraviolet cutoff imposed by the grid was higher than all physically relevant momenta in the problem. To check this we monitored the power spectra $\left|\phi_{k}\right|^{2}$ and $\left|\chi_{k}\right|^{2}$ throughout the run. On a log plot you can see that there is a momentum above which the slope of the power spectrum decreases sharply (i.e. becomes more negative), and we set our grid spacing to ensure that this cutoff was well below the cutoff of the grid. A sample spectrum for $\chi$, taken from the late stages of preheating, is shown in figure 7 . The kink that can be seen at $k \approx k_{\max } / 2$ persisted from preheating through the phase transition, and a similar feature can also be seen in the $\phi$ spectrum.

The grid spacing we used was

$$
d x=0.133 \frac{1}{\sqrt{\lambda} \phi_{0}} \approx 1.26 \times 10^{6} M_{P}^{-1}
$$

measured in comoving coordinates with $a=1$ at the beginning of the simulation. The total box size, $L=128 d x$, was equal to slightly more than 10 Hubble radii at the end of inflation.

As a further check that the ultraviolet grid cutoff was not affecting the physics

we did some smaller runs (i.e. with fewer fields) using these same parameters and another run using a grid of $64^{3}$ points with twice as large a grid spacing. Both the 


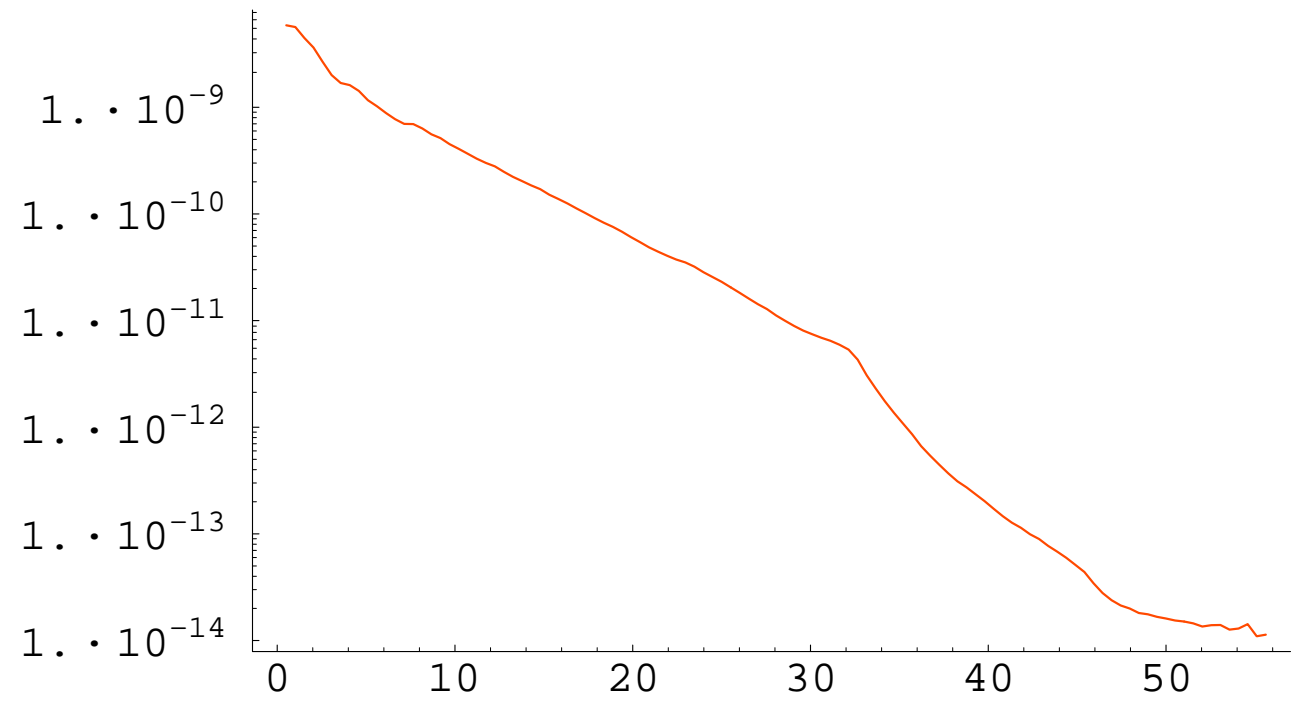

Figure 7: The spectrum $\left|\chi_{k}\right|^{2}$ as a function of momentum, shown in the late stages of preheating. The momenta $k$ are shown in units of the Hubble constant at the end of inflation.

$64^{3}$ and $128^{3}$ runs had the same total box size and they showed essentially identical behavior for the fields, suggesting that the $128^{3}$ grid has more than enough modes in the ultraviolet.

\subsection{The Field Equations}

We work in a FRW universe with metric $g_{\mu \nu}=\operatorname{diag}\left\{1,-a^{2},-a^{2},-a^{2}\right\}$. The equations of motion for the scalar fields $\phi$ and $\chi_{i}$ derive from the potential

$$
V=\frac{\lambda}{4}\left(\phi^{2}-v^{2}\right)^{2}+\frac{1}{2} g^{2} \phi^{2} \chi_{i}^{2}
$$

where $v, \lambda$, and $g$ are constant parameters and $\chi_{i}^{2}$ is understood to include a summation over $i$. The results reported here used $v=7 \times 10^{-4}, \lambda=9 \times 10^{-14}$, and $g^{2}=800 \lambda$, and took $\chi$ to be a 19-component field. This potential gives rise to the equations of motion

$$
\begin{gathered}
\ddot{\phi}+3 \frac{\dot{a}}{a} \dot{\phi}-\frac{1}{a^{2}} \nabla^{2} \phi+\left(\lambda\left(\phi^{2}-v^{2}\right)+g^{2} \chi_{i}^{2}\right) \phi=0 \\
\ddot{\chi}_{i}+3 \frac{\dot{a}}{a} \dot{\chi}_{i}-\frac{1}{a^{2}} \nabla^{2} \chi_{i}+g^{2} \phi^{2} \chi_{i}=0
\end{gathered}
$$

These equations can be simplified by the following variable redefinitions

$$
\phi_{p r}=\frac{a}{\phi_{0}} \phi ; \chi_{i, p r}=\frac{a}{\phi_{0}} \chi_{i} ; v_{p r}=\frac{1}{\phi_{0}} v ; \vec{x}_{p r}=\sqrt{\lambda} \phi_{0} \vec{x} ; \tau_{p r}=\sqrt{\lambda} \phi_{0} \int \frac{d t}{a} ; g e \equiv \frac{g^{2}}{\lambda}
$$


where $\phi_{0}$ is the initial (bare) value of the field $\phi$ and all other parameters come from the original equations of motion. These then give the rescaled equations of motion

$$
\begin{gathered}
\phi_{p r}^{\prime \prime}-\nabla_{p r}^{2} \phi_{p r}+\left(\phi_{p r}^{2}-a^{2} v_{p r}^{2}+g e \chi_{i, p r}^{2}-\frac{a^{\prime \prime}}{a}\right) \phi_{p r}=0 \\
\chi_{i, p r}^{\prime \prime}-\nabla_{p r}^{2} \chi_{i, p r}+\left(g e \phi_{p r}^{2}-\frac{a^{\prime \prime}}{a}\right) \chi_{i, p r}=0
\end{gathered}
$$

where derivatives are with respect to the rescaled time and distance variables defined above. Note that all first derivative terms have been eliminated, and the dependence on the coupling constants $\lambda$ and $g$ is now only through the ratio $g^{2} / \lambda$, denoted here by $g e$. The value of $\lambda$ itself appears only in setting the initial conditions (described below). The rescalings involving $\phi_{0}$ do not affect the equations of motion.

\subsection{The Scale Factor Equation}

Our simulations calculate a single scale factor at each time, neglecting metric fluctuations. The equation for the scale factor $a$ is derived ¿from the following two equations

$$
\begin{gathered}
\ddot{a}=-\frac{4 \pi}{3}(\rho+3 p) a \\
\left(\frac{\dot{a}}{a}\right)^{2}=\frac{8 \pi}{3} \rho
\end{gathered}
$$

where $\rho$ and $p$ are the total energy density and pressure, respectively. For simplicity we will first solve these for a single scalar field $\sigma$. The energy density and pressure can be derived ¿from the energy momentum tensor

$$
T_{\mu \nu}=\sigma_{, \mu} \sigma_{, \nu}-\frac{1}{2} g_{\mu \nu} g^{\alpha \beta} \sigma_{, \alpha} \sigma_{, \beta}+g_{\mu \nu} V(\sigma)
$$

Assuming the fields are isotropic this equation can be solved and compared to the usual energy momentum tensor of matter

$$
T_{\nu}^{\mu}=\operatorname{diag}\{\rho,-p,-p,-p\}
$$

to give

$$
\begin{aligned}
& \rho=\frac{1}{2} \dot{\sigma}^{2}+\frac{1}{2 a^{2}}|\nabla \sigma|^{2}+V(\sigma) \\
& p=\frac{1}{2} \dot{\sigma}^{2}-\frac{1}{6 a^{2}}|\nabla \sigma|^{2}-V(\sigma) .
\end{aligned}
$$

Plugging these expressions into Eq. (6.8) and using Eq. (6.9) to eliminate the $\dot{\sigma}$ term gives

$$
\ddot{a}=-2 \frac{\dot{a}^{2}}{a}+8 \pi a\left(\frac{1}{3 a^{2}}|\nabla \sigma|^{2}+V(\sigma)\right) .
$$


Switching now to the fields $\phi$ and $\chi_{i}$ and to the potential in Eq. (6.2),

$$
\ddot{a}=-2 \frac{\dot{a}^{2}}{a}+8 \pi a\left(\frac{1}{3 a^{2}}\left(|\nabla \phi|^{2}+\left|\nabla \chi_{i}\right|^{2}\right)+\frac{\lambda}{4}\left(\phi^{2}-v^{2}\right)^{2}+\frac{1}{2} g^{2} \phi^{2} \chi_{i}^{2}\right) .
$$

Finally we rescale all variables according to Eq. (6.5) and take a spatial average (denoted by " $<>$ ") over the grid

$$
a^{\prime \prime}=-\frac{a^{\prime 2}}{a}+\frac{8 \pi \phi_{0}^{2}}{a}\left\langle\frac{1}{3}\left(\left|\nabla_{p r} \phi_{p r}\right|^{2}+\left|\nabla_{p r} \chi_{i, p r}\right|^{2}\right)+\frac{1}{4}\left(\phi_{p r}^{2}-a^{2} v_{p r}^{2}\right)^{2}+\frac{1}{2} g e \phi_{p r}^{2} \chi_{i, p r}^{2}\right\rangle .
$$

\subsection{Initial Conditions}

Although the field equations are solved in configuration space with each lattice point representing a position in space, the initial conditions are set in momentum space and then Fourier transformed to give the initial values of the fields and their derivatives at each grid point. It is assumed that no significant particle production has occurred before the beginning of the program, so Minkowski space quantum fluctuations are used for setting the initial values of the modes. ${ }^{1}$

The calculation of these modes is given in detail in [15]. The result is that each mode $\sigma_{k}$ has a probability distribution given by

$$
P\left(\sigma_{k}, \sigma_{k}^{*}\right) \propto e^{-2 \omega_{k} \sigma_{k} \sigma_{k}^{*}}
$$

where $\omega_{k} \equiv \sqrt{k^{2}+m^{2}}$. Separating $\sigma_{k}$ into a magnitude and a phase, the phase has a uniform probability distribution and the magnitude has a Rayleigh distribution

$$
P\left(\left|\sigma_{k}\right|\right) \propto\left|\sigma_{k}\right| e^{-2 \omega_{k}\left|\sigma_{k}\right|^{2}} .
$$

The momentum $k$ is simply the Fourier transform variable and the mass is given by

$$
m_{\sigma}^{2}=\frac{\partial^{2} V}{\partial \sigma^{2}} \approx\left\{\begin{array}{l}
3 \lambda \phi_{0}^{2}, \phi \\
g^{2} \phi_{0}^{2}, \chi_{i}
\end{array}\right.
$$

Taking into account the finite size of the box, $L$, and the discretization of the spatial points with spacing $d x$ and converting to the rescaled variables defined above yields for the initial magnitudes

$$
\left|\phi_{k, p r}\right|=\frac{\sqrt{\lambda} L_{p r}^{3 / 2}}{\sqrt{2} d x_{p r}^{3}\left(k_{p r}^{2}+3\right)^{1 / 4}}
$$

\footnotetext{
${ }^{1}$ This approximation is usually very good for fluctuations with momenta greater than $H$. For smaller momenta one can find a better approximation after performing the field quantization in curved space, see e.g. [24], but in our case the corresponding corrections only slightly modify the final result.
} 


$$
\left|\chi_{i k, p r}\right|=\frac{\sqrt{\lambda} L_{p r}^{3 / 2}}{\sqrt{2} d x_{p r}^{3}\left(k_{p r}^{2}+g e\right)^{1 / 4}}
$$

times a Rayleigh distributed random number with standard deviation 1 . Note that the program values of $L, d x$, and $k$ are defined by the rescaling of $x$, and recall that $g e \equiv g^{2} / \lambda$. Finally the zero mode, which appears as a value uniformly added to all grid points at the beginning of the calculation, is set to 0 for $\chi_{i}$ and 1 for $\phi$ (since $\left.\phi_{p r}=\phi / \phi_{0}\right)$.

In order to set the field derivatives it is necessary to know the time dependence of the vacuum fluctuations being considered. In Minkowski space this time dependence is given simply by the term $e^{-i \omega t}$ which suggests

$$
\dot{\sigma}_{k}=-i \omega_{k} \sigma_{k}
$$

Converting to rescaled values of time, mass, and momentum gives

$$
\begin{gathered}
\phi_{k, p r}^{\prime}=i \phi_{k, p r} \sqrt{k_{p r}^{2}+3} \\
\chi_{i k, p r}^{\prime}=i \chi_{i k, p r} \sqrt{k_{p r}^{2}+g e}
\end{gathered}
$$

The initial derivatives of the zero modes of $\phi$ and $\chi_{i}$ are set to 0 .

Note that having been eliminated from the equations of motion, the only place $\lambda$ shows up in the calculations at all aside from the ratio $g^{2} / \lambda$ is in the $\sqrt{\lambda}$ term in the magnitude of the initial fluctuations. The coupling constant $g$ appears nowhere except in $g^{2} / \lambda$. Meanwhile the initial value of the field $\phi$, i.e. $\phi_{0}$, is used in the rescaling of the field and spacetime variables but it appears neither in the equations of motion for the fields nor in the amplitude of their initial fluctuations. In fact it appears in two places in the calculation. The first is in the evolution equation for the scale factor, Eq. (6.16). The second way the initial value of $\phi$ enters the calculations is subtler. We said that the zero mode of $\phi_{p r}^{\prime}$ is initially set to 0 . At first this may seem like a poor choice since the field $\phi$ initially must be rolling towards 0 . In fact the beginning of the program is supposed to represent the end of inflation when the slow roll approximation is no longer valid, so why should $\phi_{p r}^{\prime}$ be set to 0 ? The answer comes from the use of the conformal field $\phi_{p r} \propto a \phi$. As time goes on $\phi$ is decreasing but $a$ is increasing, and there is a moment when these two balance and $\phi_{p r}^{\prime}$ is momentarily 0 . By setting $\phi_{p r}^{\prime}=0$ the program automatically sets the beginning of the calculation at this moment. For the potential described here this moment occurs when $\phi \approx .35 M_{p}$, so the initial conditions implicitly use the value of $\phi_{0}$. In the one place in the program where it does appear (in the scale factor evolution) this parameter is set to .35. This value is also useful in converting the output of the program to physically meaningful units. 


\subsection{The Calculational Method: Staggered Leapfrog}

The differential equations derived above are solved using a staggered leapfrog algorithm in which the field values and their time derivatives are calculated at alternating times separated by a half time-step. The time step is kept constant throughout the calculation. (Since the program uses conformal time the physical time elapsed during each time step changes as the program progresses.) This method is stable for second order equations involving no first time derivatives such as the field equations we use, see e.g. [25]. However, extra care must be taken in solving the equation for the scale factor, Eq. (6.16), since this does contain a first derivative term $a^{\prime}$. A naive calculation using the leapfrog algorithm as described above would mean that $a^{\prime \prime}$ would be calculated at time $\tau$ as a function of $a(\tau)$ and $a^{\prime}(\tau-d \tau / 2)$. There is a solution for this, although in practice the evolution of $a$ is so slow and smooth that this problem makes no practical difference. We avoid this problem, though, by using the two following equations ${ }^{2}$

$$
\begin{aligned}
& a^{\prime}(\tau+d \tau / 2) \approx a^{\prime}(\tau-d \tau / 2)+d \tau a^{\prime \prime}(\tau) \\
& a^{\prime}(\tau) \approx \frac{1}{2}\left(a^{\prime}(\tau+d \tau / 2)+a^{\prime}(\tau-d \tau / 2)\right) .
\end{aligned}
$$

Solving these simultaneously, using Eq. (6.16) for $a^{\prime \prime}$, gives

$$
a^{\prime}(t+d \tau / 2) \approx-a^{\prime}(\tau-d \tau / 2)+\frac{2 a}{d \tau}\left(-1+\sqrt{1+2 \frac{a^{\prime}(\tau-d \tau / 2)}{a} d \tau+\frac{f}{a} d \tau^{2}}\right)
$$

where

$$
f(a(\tau))=\frac{8 \pi \phi_{0}^{2}}{a}\left\langle\frac{1}{3}\left(\left|\nabla \phi_{p r}\right|^{2}+\left|\nabla \chi_{i, p r}\right|^{2}\right)+\frac{1}{4}\left(\phi_{p r}^{2}-a^{2} v_{p r}^{2}\right)^{2}+\frac{1}{2} g e \phi_{p r}^{2} \chi_{i, p r}^{2}\right\rangle .
$$

Since Eq. (6.16) needs to be solved only once per time step this correction involves virtually no added computational time.

\footnotetext{
${ }^{2}$ G.F. would like to thank Julian Borrill, who suggested this solution.
} 


\section{References}

[1] D.A. Kirzhnits, JETP Lett. 15, 529 (1972); D.A. Kirzhnits and A.D. Linde, Phys. Lett. 42B, 471 (1972).

[2] S. Weinberg, Phys. Rev. D9, 3320 (1974); L. Dolan and R. Jackiw, Phys. Rev. D9, 3357 (1974); D.A. Kirzhnits and A.D. Linde, Sov. Phys. JETP 40, 628 (1974).

[3] D.A. Kirzhnits and A.D. Linde, Ann. Phys. 101, 195 (1976).

[4] A. D. Linde, Particle Physics and Inflationary Cosmology (Harwood, Chur, Switzerland, 1990)

[5] A.H. Guth, Phys. Rev. D23, 347 (1981); A.D. Linde, Phys. Lett. 108B, 389 (1982);

A. Albrecht and P.J. Steinhardt, Phys. Rev. Lett. 48, 1220 (1982).

[6] V.A. Kuzmin, V.A. Rubakov and M.E.Shaposhnikov, Phys. Lett. 155B (1985) 36;

M.E. Shaposhnikov, JETP Lett. 44 (1986) 465; Nucl. Phys. B287 (1987) 757; Nucl. Phys. B299 (1988) 797.

[7] L. Kofman, A. Linde, and A. A. Starobinsky, Phys. Rev. Lett. 73, 3195 (1994); L. Kofman, A. Linde, and A. A. Starobinsky, Phys. Rev. D 56, 3258 (1997).

[8] L. Kofman, A. Linde, and A. A. Starobinsky, Phys. Rev. Lett. 76, 1011 (1996).

[9] I. I. Tkachev, Phys. Lett. B376, 35 (1996).

[10] S. Khlebnikov, L. Kofman, A. Linde, and I. Tkachev, Phys. Rev. Lett. 81, 2012 (1998), hep-ph/9804425.

[11] A. Rajantie and E. J. Copeland, "Phase transitions from preheating in gauge theories," hep-ph/0003025.

[12] D.H. Lyth and E.D. Stewart, Phys. Rev. Lett. 75, 201 (1995).

[13] I. Tkachev, S. Khlebnikov, L. Kofman, and A. Linde, Phys. Lett. B440, 262-268 (1998), hep-ph/9805209.

[14] S. Kasuya, M. Kawasaki, Phys. Rev. D61, 083510 (2000), hep-ph/9903324.

[15] S. Khlebnikov, and I. Tkachev, Phys. Rev. Lett. 77, 219 (1996), hep-ph/9603378; S. Khlebnikov and I. I. Tkachev, Phys. Rev. Lett. 79, 1607 (1997).

[16] E. W. Kolb and M. S. Turner, The Early Universe (Addison-Wesley, Redwood City, California, 1990).

[17] A.D. Linde, Nucl. Phys. B372, 421 (1992).

[18] S. Coleman, Phys. Rev. D15 (1977) 2929.

[19] A.D. Linde, Nucl.Phys. B216, 421 (1983). 
[20] Ya.B. Zeldovich, I.Yu. Kobzarev, and L.B. Okun, Phys. Lett. 50B, 340 (1974).

[21] A.D. Linde and A. Riotto, Phys. Rev. D 56, 1841 (1997), hep-ph/9703209; C. Contaldi, M. Hindmarsh, J. Magueijo, Phys. Rev. Lett. 82, 2034 (1999), astro-ph/9809053; R.A. Battye, J. Weller, Phys. Rev. D61, 043501 (2000), astro-ph/9810203; R. A. Battye, J. Magueijo, J. Weller, astro-ph/9906093.

[22] R. Kallosh, L. Kofman, A. Linde and A. Van Proeyen, "Gravitino production after inflation," hep-th/9907124.

[23] G. F. Giudice, I. Tkachev, and A. Riotto, JHEP 9908, 009 (1999), hep-ph/9907510.

[24] G. Felder, L. Kofman, and A. Linde, "Gravitational particle production and the moduli problem," JHEP, 02, 027 (2000), hep-ph/9909508.

[25] K.C.B. New, K. Watt, and C.W. Centrella, Stable Three Level Leapfrog Integration in Numerical Relativity, Phys. Rev. D58, 064022 (1998), gr-qc/9801110. 